\title{
An Experiment on Fear of Public Speaking in Virtual Reality
}

\author{
D-P. Pertaub ${ }^{1}$, M. Slater ${ }^{1}$ and C. Barker ${ }^{2}$ \\ Department of Computer Science, University College London, Gower Street, \\ WC1E 6BT, United Kingdom ${ }^{1}$
}

Department of Psychology, University College London ${ }^{2}$

\begin{abstract}
Can virtual reality exposure therapy be used to treat people with social phobia? To answer this question it is vital to know if people will respond to virtual humans (avatars) in a virtual social setting in the same way they would to real humans. If someone is extremely anxious with real people, will they also be anxious when faced with simulated people, despite knowing that the avatars are computer generated? In [17] we described a small pilot study that placed 10 people before a virtual audience. The purpose was to assess the extent to which social anxiety, specifically fear of public speaking, was induced by the virtual audience and the extent of influence of degree of immersion (head mounted display or desktop monitor. The current paper describes a follow up study conducted with 40 subjects and the results clearly show that not only is social anxiety induced by the audience, but the degree of anxiety experienced is directly related to the type of virtual audience feedback the speaker receives. In particular, a hostile negative audience scenario was found to generate strong affect in speakers, regardless of whether or not they normally suffered from fear of public speaking.
\end{abstract}

\section{Introduction}

Social phobia is a prevalent and disabling anxiety disorder [1]. People who suffer from social phobia fear, and if possible avoid, one or more social performance situations. One common example of social phobia is fear of public speaking. This can be particularly difficult for the sufferer as presentation giving is crucial to many responsible jobs and thus fear of public speaking can have a serious impact on an individual's career prospects.

Cognitive-behaviour therapy is the leading psychological treatment for phobias. It typically involves systematic exposure to feared situations ('exposure therapy') and the use of cognitive techniques to help patients identify and challenge anxiety-related negative thoughts. Avoidance behaviour is overcome in the course of the exposure sessions, where patients note that their anxiety attenuates with prolonged exposure to the stimulus, a process known as habituation.

Virtual reality can be used in exposure therapy to substitute an artificially created and controlled stimulus for the real one. An increasing number of case studies and anecdotal reports claim success for treating a wide range of specific phobias, such as fear of heights, fear of flying and fear of spiders $[2,3,4,5,6,7,8]$. While there have been many published studies of the application of virtual reality to specific phobias, to our knowledge there is only one concerning social phobias [9]. In contrast to that study, 
which used a virtual lecture theatre with a large audience, we concentrated on a small seminar type setting. Rather than conducting therapy, we were interested in the prior question of whether a speaker's anxiety response depends on the type of feedback received from a virtual audience. If appropriate affect is generated by the experience of speaking to a small group of avatars, it would suggest that virtual reality can be successfully deployed in the treatment of other social phobias. Studying small groups will help us to understand the design parameters for creating successful synthetic social encounters.

Three different virtual audiences were developed (negative, positive and static) and an experiment was conducted to explore whether the type of audience would influence the emotional response of the speaker. Details of the experiment are described in Section 2. A summary of the between-groups results and the written comments as well as a report of the debriefing sessions is given in Section 4. Conclusions are presented in Section 5. A more in-depth description of the experiment and formal statistical results are left to another paper [10].

\section{Experiment}

\subsection{Experiment Design}

Forty three subjects were recruited for the experiment, but three sets of results were discarded because of incomplete data. All subjects gave at least two talks to a virtual audience. Audience type was one factor in the experiment. Subjects were randomly assigned to one of three groups, distinguished by the type of virtual audience they spoke to first. Subjects who gave their first talk to either the negative or the positive audience gave their second talk to the other animated audience. Subjects who spoke to the static audience gave their second talk to the static audience again. The second factor was immersion, or the mode of delivery of the virtual environment (full stereo headmounted display or desktop monitor).

If only the results of the first talk are considered, the design is a between-groups experiment. All the results together can be thought of as a within-groups design.

\subsection{Scenario}

The virtual audience consisted of a group of eight formally dressed male avatars, seated in a semicircle around a table. In order to foster the illusion of life, the avatars continuously exhibited small twitching movements, blinking and shifting about in their chairs. Two animated audience scenarios were scripted, designed to model the behaviour and reactions of a friendly, appreciative audience and a hostile, unreceptive audience. Facial animations, direction of gaze, body posture and short animations were selected to convey as unambiguous an evaluative message as possible, and the choice of responses was aided by reference to the literature on non-verbal communication $[11,12,13]$. Each audience scenario consisted of a set of ten major audience responses. The distributive properties of the DIVE virtual environment [14] were exploited to allow an unseen operator to listen to the talks and trigger the next response in the sequence at appropriate points in the speech. The operator could also play a selection of audio recordings of audience comments at any time during the talk. 

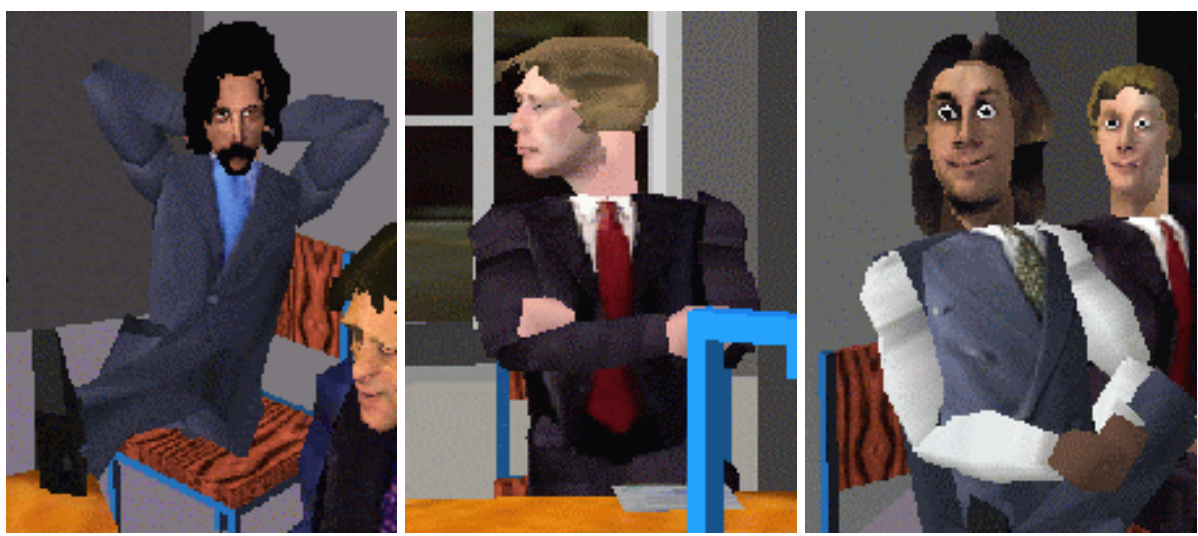

Figure 1. Various postures and facial expressions communicate listener attitudes. From left to right: an audience member puts his feet on the table with arms behind head indicating extreme relation and dominance. Closed upper arm posture communicates disagreement [11] while large smiles and plenty of eye contact suggest interest and an affinity for the speaker [12]. Crossed legs do not consistently convey either positive or negative attitudes to the speaker.

\subsection{Method}

Subjects were students or members of staff at University College London. Each subject came into the Department of Computer Science twice, with an interval of a couple of days between the visits. On the first occasion, subjects completed the Personal Report of Confidence as a Speaker (PRCS) [15] and the Fear of Negative Evaluation [16] questionnaires. The PRCS provides a measure of general public speaking anxiety. Subjects were instructed to prepare a five minute talk for presentation at the next visit. They were told they would give the same presentation twice, to a computer generated virtual audience. They were informed that the talk might be recorded for later analysis. No details of the workings of the system were provided.

On the day of the talks, subjects using the head mounted display stood in an empty darkened room while giving their talk. Subjects using the 21 inch desktop monitor sat alone in the same darkened room. All subjects were fitted with a microphone and head-phones. A concealed operator sat at a remote terminal and played a pre-recorded invitation to begin speaking. At appropriate intervals, the operator triggered the next in a pre-determined sequence of ten animated audience responses. In the case of the static audience, the operator simply monitored the talk. A warning was played 30 seconds from the end and an audio clip told the speaker to stop after 5 minutes. At the end of each talk, subjects were taken to a different room and asked to complete questionnaires asking them about the experience.

All subjects gave at least two talks. Subjects who gave their second talk to the negative audience were asked to give a short third talk, this time to the positive audience again. For ethical reasons we wanted the last experience to be a pleasant one. No data was collected for this talk.

\section{Variables}

There were three main response variables, two designed to assess the degree of self-reported anxiety generated by the experience of giving the talk and another to measure the speaker's assessment of their performance. The first was a modified form of the Personal Report of Confidence of a Public Speaker (MPRCS), altered to refer to the talk just given. A checklist of 9 anxiety symptoms (sweating, discomfort in stomach, heart palpitations, tremors, nerves/feelings of being scared, tightness in chest, tenseness, 
loss of balance, tenseness) provided a second response variable (Somatic). The third variable (self-rating) was the subject's own assessment of their performance, rated on a scale from 0 to 100 where 0 indicated complete dissatisfaction and 100 indicated complete satisfaction with their talk.

\section{Results}

\subsection{Statistical Results.}

The results from the between groups design are discussed in depth in [10]. A short summary for each of the main response variables of the main findings follows.

Modified PRCS. The MPRCS is a measure of the anxiety experienced by the subject when speaking to the virtual audience. A logistic regression analysis was performed on the data for post-talk MPRCS. The best fitting regression model found included the two main factors (immersion, audience type) and positive correlation between the PRCS measured prior to all talks and MPRCS, measured after the talk. The group that spoke to the negative audience had a significantly higher MPRCS than the other groups (positive or static audiences). Interestingly, the slope of the regression line of MRPCS on prior PRCS was significantly different between the audience types. For the positive and static audiences there was a positive slope, but for the negative audience the slope was not significantly different from 0 . This means that the prior PRCS cannot predict the post PRCS when the audience faced by the subject was negative.

Somatic and Self-Rating. For the response variable Somatic, a logistic regression analysis found only audience type and prior PRCS to be significant. The negative audience group exhibited by far the highest level of somatic response, while the positive and static audience groups had lower response levels with no significant difference between them. A normal regression model was performed on self-rating and both prior PRCS and audience type were significant. There was no significant difference between positive and negative audience results for self rating. Self rating would appear to be lower if the audience is animated.

\subsection{Debriefing session and written reports}

After each talk, subjects were asked to write down any comments about the talk they had just given, and were prompted to mention the best and worst aspects of the speech. At the end of the experiment, subjects were invited to expand upon these comments and draw comparisons between the different audience conditions they had experienced. These debriefing sessions afforded us an opportunity to obtain a deeper and richer picture of the sessions than was possible with the written questionnaire entries.

It was clear from the outset that almost all subjects were treating the experiment as a serious opportunity to give a talk. Their assumption was that their role was to try and win the audience over, a plan that seemed reasonable despite the fact they were facing a virtual audience.

\subsubsection{Animated audience group comments}

For the most part, the affect generated was appropriate to the type of audience response, but there were clearly some idiosyncratic reactions amongst the subjects. All subjects speaking to an animated audience were successfully able to decode the valence 
of the audience response. Due to the stereotyped nature of some of the responses, several subjects (7) reported a sense of amusement when the audience reacted, although a number of these subjects admitted that the amusement was paired with feelings of tension and nervousness.

Twelve subjects reported that although they knew the audiences were not real, the experiences felt like giving a real presentation in many significant respects. Similarities included heightened anxiety just before the talks, emotional reactions to the audience while speaking and the use of presentation strategies to attempt to connect with the audience. Subjects made the following comments:

'You get involved in a situation, it doesn't matter whether it's real or not. I needed to try to convince them.'

'The emotions were similar. Before I gave the talk I felt exactly as I would feel in the department. I had cold hands and I was sweating!'

'It felt a lot like a presentation, like the ones that I give in the department. I approached it in the same way as a real presentation. I jotted down notes the night before..'.

The negative audience was capable of generating very strong speaker emotions, regardless of whether it was encountered at the first talk or at the second. Subjects repeatedly reported they could find no way to 'bring back' the audience when the avatar listeners lost interest. Some subjects interpreted their incapacity to influence the audience as a failure on their part as a speaker. Many reported feelings of confusion or disorientation and a tendency to forget what they wanted to say. Six subjects described the experience as very disturbing or disconcerting. Five subjects commented they would have walked out of the room if they had received a response like that from a real audience. One subject mentioned that it was immensely satisfying to be able to survive the audience. Another confided that the thought 'My talk is boring' kept reverberating inside his head. Other subjects commented:

'It felt really bad. I couldn't just ignore them. I had to talk to them and tell them to sit up and pay attention. Especially the man on the left who put his head in his hands; I had to ask him to sit up and listen.... I entered a negative feedback loop where I would receive bad responses from the audience and my performance would get even worse.... I was performing really badly and that doesn't normally happen.'

'I was upset, really thrown. I totally lost my train of thought. They weren't looking at me and I didn't know what to do. Should I start again? I was very frustrated. I felt I had no connection to them. They weren't looking at me. I just forgot what I was talking about.'

'The first audience was terrible. They were really disturbing. From the beginning they were uninterested and it really put you off..... I couldn't say everything I wanted to say.'

The pattern of subject response to the positive audience scenario was much less homogenous. Some subjects clearly took heart from the enthusiastic response of their listeners. One subject recalled 'It was clear that one audience was really positive and interested in what I was saying and it made you feel like telling them what you know.' Another noted 'I felt great. Finally nobody was interrupting me. Being a woman, people keep interrupting you in talks much more... But here I felt people were there to listen to me.' One commented 'They were staring at me. They loved you unconditionally, you could say anything, you didn't have to work'. However nine subjects felt that the positive audience responses were very exaggerated and became off-putting and distracting. Two commented that it lacked the challenge and the audience 'buzz' of the negative scenario.

Ten people explicitly claimed that the negative audience was the more realistic of the two. One subject summed up this view when she said 'I found the first (positive) 
audience fairly unconvincing; they all sat up straight and stared at me with these moronic grins and made comments like 'wonderful' at inappropriate moments'. Six subjects stressed that the timing of audience responses seemed to be a determining factor. One explained that it was very off-putting to be congratulated when they began to make an important point, rather than after it.

\subsubsection{Static audience group comments}

All subjects speaking to the static audience commented on how stiff and unresponsive the audience was. This was especially the case with the desktop condition, where subjects talked to what was effectively a static image of an audience. One subject reported that 'it was more like talking to a mirror than to a real audience. There was no sense of satisfaction because there was no feedback, I didn't know if they liked it or not... It felt like a real practice presentation. I tend to practice speaking to a wall. This was slightly more real'. Subjects also had a tendency to be more critical of the appearance of the avatars; the subject's written responses for the static audience were full of suggestions on how the look of the computer characters could be improved. Most subjects felt that their second talk was better than the first, perhaps unsurprising as they were giving the same talk twice to the same static audience.

\subsubsection{Discussion}

Both the statistical results and the debriefing sessions clearly show the anxiety provoking effect of the negative audience scenario. An intriguing result is the claim that the negative audience was more realistic than the positive one. Both audiences featured exaggerated reactions and were subject to the same delays in triggering responses. But in real seminar settings, encouraging comments and gestures tend to occur at very specific points during a talk - the end of phrases, units of information or other characteristic segments of speech. Very short response intervals govern these feedback mechanisms. The negative audience did not listen and did not have to obey these conversation facilitating conventions. It was realistic but at the same time unusual: In many societies, there tend to be few genuine overt displays of negative evaluation in social exchanges between adults [12]. That might account for part of the reason why the negative audience was found quite so disturbing.

\section{Conclusions}

These results confirm the results of our initial pilot study [16]. People responded to virtual seminar audiences much as they would respond to real audiences; they felt more at ease with a positive group of listeners and experienced considerable discomfort with an unpleasant, unforgiving audience. With the animated audience scenarios, and with the negative audience scenario in particular, there was sufficient co-presence to elicit strong affect in virtual speakers. Our findings indicate that virtual reality scenarios with computer generated characters could be of use in treating and investigating a range of social performance situations, but that the appropriate timing of avatar responses is critical to maintaining co-presence. 


\section{References}

[1] Kessler, R.C., McGonagle, K.A., Zhao, S., Nelson, C.B., Hughes, M., Eshleman, S., Wittchen, H. U., and Kendler, K.S. (1994) Lifetime and 12 -month prevalence of DSM-III-R Psychiatric Disorders in the United States, Archives of General Psychiatry, 51, 8-19.

[2] Botella, C., Banos, R. M., Perpina, C., Villa, H., Alcaniz, M., Rey, A. (1998) Virtual Reality Treatment of Claustrophobia. Behaviour Research and Therapy 36(2):239 -246, February 1998.

[3] Carlin, A., Hoffman, H. G., Weghorst, S., (1997) Virtual reality and tactile augmentation in the treatment of spider phobia: a case report. Behaviour Research and Therapy 35, 153 -158.

[4] North, M. M., North S. M., Coble, J.R., (1998) Virtual Reality Therapy: An effective treatment for phobias, Virtual Environments in Clinical Psychology and Neuroscience, Stu dies in Health Technology and Informatics, Volume 58.

[5] Rothbaum, B. O., Hodges, L. F., Kooper, R., Opdyke, D., Williford, J., North, M. M., (1995a) Effectiveness of Computer-Generated (Virtual Reality) Graded Exposure in the Treatment of Acrophobia. American Journal of Psychiatry, 152, 626-628.

[6] Rothbaum, B. O., Hodges, L. F., Kooper, R., Opdyke, D., Williford, J., (1995b). Virtual reality graded exposure in the treatment of acrophobia: a case study. Behaviour Therapy. 26, 547 -554.

[7] Rothbaum, B. O., Hodges, L. F., Watson, B. A., Kessler, G. S., Opdyke, D. (1996) Virtual reality exposure therapy in the treatment of fear of flying: a case report. Behaviour Research and Therapy. 34, 477-481.

[8] Strickland, D., Hodges, L., North, M., Weghorst, S., (1 997) Overcoming Phobias by Virtual Exposure, Communications of the ACM, volume 40, p 34-40.

[9] North, M. M., North S. M., Coble, J.R., (1998) Virtual Reality Therapy: An effective treatment for the fear of public speaking. International Journal of Virtua 1 Reality Vol.3 No.2, 2-6.

[10] Pertaub, D-P., Slater, M., Barker, C., (2000) An experiment on public speaking anxiety in response to three difference types of virtual audience.

[11] Bull, P. (1987), Posture and Gesture, Oxford, Pergamon.

[12] Argyle, M. (1988) Bodily Communication, Methuen \& Co Ltd .

[13] Mehrabian, A., (1968) Relationship of attitude to seated posture, orientation and distance. Journal of Consulting and Clinical Psychology 32:296-308.

[14] Frecon, E. and Stenius, M. (1998) DIVE: A scal eable network architecture for distributed virtual environments, Distributed Systems Engineering Journal, 5(3), 91 -100.

[15] Paul, G. (1966) Insight vs. desensitization in psychotherapy. Stanford University Press.

[16] Watson, D., Friend, R., (1969) Measur ement of social-evaluative anxiety (FNE), Journal of Consulting and Clinical Psychology 33 448-457.

[17] Slater, M., Pertaub, D-P., and A.Steed (1999) Public Speaking in Virtual Reality: Facing and Audience of Avatars, IEEE Computer Graphics and Applicat ions, 19(2), March/April 1999, p6-9. 